\title{
MORPHOLOGIE ET CHÉTOTAXIE D'UNE CERCAIRE DE SPIRORCHIIDAE DU TOGO
}

\author{
Ch. BAYSSADE-DUFOUR *, S. D. KULO **, J.-L. ALBARET *
}

RÉSUMÉ. Des cercaires distomes, apharyngées, brévifurquées, ocellées, pourvues d'une crête dorsale réduite, de palettes natatoires au niveau des fourchons à l'extrémité desquels s'ouvrent les pores excréteurs, sont émises par un Bulinus globosus naturellement infesté du Togo. Issues de sporocystes, elles présentent tous les caractères des cercaires de Spirorchiidae.

C'est la première mention de telles cercaires en Afrique. La chétotaxie étudiée pour la première fois chez cette famille montre d'importantes différences avec celles des Schistosomatidae et met en évidence quelques analogies avec celle de Transversotrema patialense (Azygiata: Transversotrematidae).

Mots-clés : Digenea. Spirorchiidae. Cercaires. Morphologie. Chétotaxie. Togo. Bulinus globosus.

\section{Morphology and chaetotaxy of the cercaria of a Spirorchiidae from Togo.}

SUMMARY. Distomatous, apharyngeate, brevifurcate, ocellate, dorsally crested cercariae, with tail furcae provided with finfolds and an excretory pore at their tip, were shed by a wild Bulinus globosus from Togo. They develop from sporocysts and display the characteristics of cercariae of Spirorchiidae. This is the first mention of such cercariae in Africa and the first chaetotaxic description of a member of this family. The chaetotaxy shows important differences with the Schistosomatidae and some similarities with that of Transversotrema patialense (Azygiata: Transversotrematidae).

Key-words: Digenea. Spirorchiidae. Cercaria. Morphology. Chaetotaxy. Togo. Bulinus globosus.

\section{Introduction}

Les Spirorchiidae sont, à de rares exceptions près, hébergés par des Chéloniens. Ces Trématodes parasites de l'appareil circulatoire sont particulièrement répandus en Amérique du Nord et en Asie, où ils ont été observés de longue date (Skrjabin, 1951 ; Smith, 1972 ; Yamaguti, 1971 et 1975). Les adultes n'ont été

* Laboratoire de Zoologie-Vers, associé au CNRS, Muséum National d'Histoire Naturelle, 61, rue Buffon, F 75231 Paris Cedex 05.

** Laboratoire de Zoologie, Université du Bénin, B. P. 1515, Lomé, Togo.

Accepté le 4 janvier 1989. 
que rarement signalés ou décrits sur le continent africain (Looss, 1899 ; Justine, 1985; Bourgat et Kulo, 1987 ; Goodman, 1987) et aucune cercaire, à notre connaissance, n’y a été signalée jusqu'à ce jour.

Ayant eu l'occasion de disposer de cercaires émises par un Bulinus globosus (Morelet) du Togo et, bien que leur appartenance à une espèce déterminée n'ait pas été établie, nous avons pensé qu'il était intéressant d'étudier leur chétotaxie puisque l'ensemble de leurs caractères morphologiques les rattachent indiscutablement aux Spirorchiidae et que la chétotaxie des cercaires de cette famille, inconnue jusqu'à présent, permet la comparaison avec celle d'autres furcocercaires appar-tenant aux Strigeata et Azygiata.

\section{Matériel et méthode}

Les cercaires proviennent d'un $B$. globosus naturellement infesté, récolté le 2 mars 1988 à Panassimkopé, près de Kolokopé, dans la région d'Atakpamé, Togo.

Elles sont émises, après exposition du Mollusque, à la lumière. Leur organisation interne et externe est mise en évidence par diverses méthodes : dessins des cercaires vivantes ou après fixation au formol à $10 \%$, coloration au Carmin chlorhydrique alcoolique, imprégnation au protéinate d'argent par la technique de Bodian modifiée par Dragesco et Djiné in Albaret (1973).

Leurs récepteurs sensoriels sont imprégnés au nitrate d'argent par la méthode de Combes et coll. (1976).

Les cercaires prennent naissance dans des sporocystes ovoïdes à paroi très fine.

L'essai d'infestation d'une jeune Tortue de Floride, Chrysemis picta, a échoué. Ceci peut être dû au nombre réduit de cercaires utilisées ou au fait de l'absence de permissivité d'une Tortue américaine à un parasite africain.

L'identification Spirorchiidae est donc donnée d'après les caractéristiques morphologiques des cercaires telles qu'elles sont précisées par Skrjabin (1951), Smith (1972), Yamaguti (1975) et Nassi (1980).

\section{Morphologie des cercaires}

Les mesures portent sur 6 cercaires fixées au formol chaud à $10 \%$.

Longueur du corps cercarien : $165 \mu \mathrm{m}(150 \mu \mathrm{m}$ à $178 \mu \mathrm{m})$.

Longueur du tronc caudal $: 460 \mu \mathrm{m}(440 \mu \mathrm{m}$ à $482 \mu \mathrm{m})$.

Longueur des fourchons $\quad: 170 \mu \mathrm{m}(168 \mu \mathrm{m}$ à $178 \mu \mathrm{m})$.

\section{Organisation GÉnÉrale (fig. 1)}

-- La cercaire ne possède pas de ventouse orale, mais un organe de pénétration piriforme, extensible, couvert antérieurement de fines épines.

- L'acétabulum post-équatorial est petit et proéminent. 

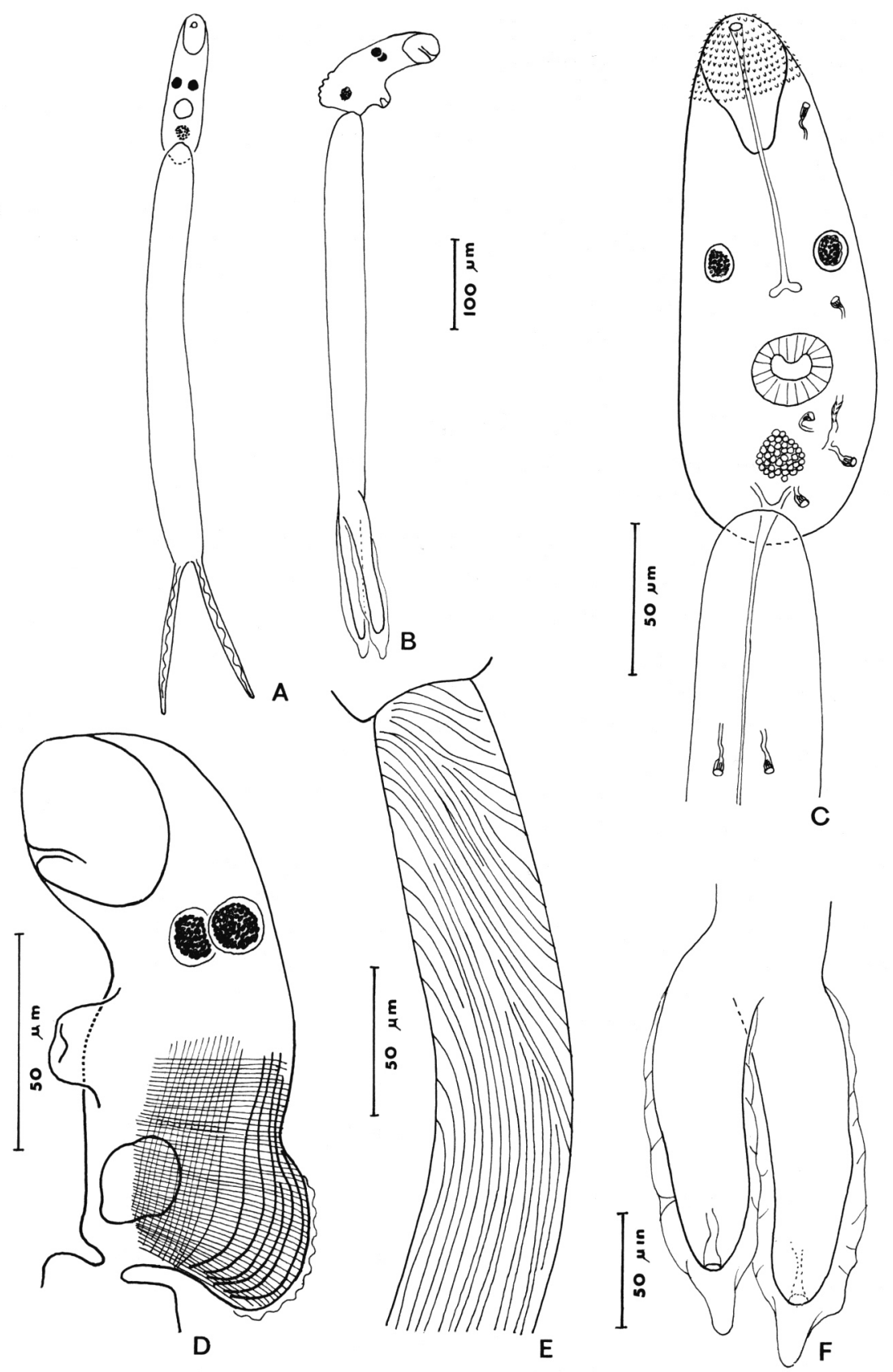

Fig. 1. - A et B : aspect général de la cercaire. A, vue ventrale; $\mathrm{B}$, vue latérale. $\mathrm{C}$ : Morphologie générale du corps cercarien et de la région proximale du tronc caudal, vue ventrale. $\mathrm{D}$ : vue latérale, musculature de la région postérieure. $\mathrm{E}:$ musculature du tronc caudal. $\mathrm{F}$ : détail des fourchons. 
- La bouche s'ouvre ventralement dans l'organe de pénétration, suivie d'un long œsophage et de deux courts cæcums.

- La vessie bilobée reçoit les produits d'excrétion véhiculés par deux canaux, l'un draînant la région antérieure, l'autre la région postérieure du corps ; la portion de canal située sous l'acétabulum contient un pinceau interne de cils vibratiles; le canal excréteur caudal parcourt tout le tronc caudal ; juste avant la bifurcation distale, il se dilate en un réservoir qui se remplit et se vide régulièrement, puis il se divise en deux branches, une dans chaque fourchon. Un pore excréteur s'ouvre à l'extrémité de chaque fourchon.

Le corps contient 5 paires de cellules flammes et la queue 1 paire dans sa région proximale, soit :

$$
2[(1+1)+(1+1+1+[1])]=12 .
$$

- Les glandes de pénétration situées dorsalement dans la moitié postérieure n'ont pu être dénombrées.

- L’ébauche génitale s'observe entre l'acétabulum et la vessie.

- Les 2 ocelles situés vers l'arrière de la moitié antérieure du corps sont composés d'une partie pigmentée granuleuse, surmontée d'un disque transparent.

- La partie postérieure du corps porte une proéminence dorsale très musclée, surmontée d'une courte crête.

Le tronc caudal est fixé ventralement sur le corps un peu en arrière de l'acétabulum ; il est doté d'une forte musculature : les fibres musculaires proximales sont obliques, les autres sont parallèles aux bords du tronc caudal. Les fourchons caudaux portent une nageoire ventro-dorsale.

Par l'ensemble de leurs caractères, ces cercaires appartiennent à la famille des Spirorchiidae.

Notons qu'il s'agit de la première mention d'une cercaire de cette famille en Afrique et chez un Bulinus; les cercaires de Spirorchiidae déjà décrites parasitent des Mollusques appartenant aux genres Helisoma, Ferrissia, Menetus ou Physa, en Amérique, et Indoplanorbis, en Asie, selon Nassi (1980).

\section{L'appareil sensoriel}

$a$ - LA RÉGion CÉPHALiQue (fig. 2)

Les papilles de la région céphalique sont disposées selon quatre cycles, soit par hémicorps :

$\mathrm{C}_{\mathrm{I}}=1 \mathrm{C}_{\mathbf{I}} \mathrm{V}, 3 \mathrm{C}_{\mathbf{I}} \mathrm{L}, 4 \mathrm{C}_{\mathbf{I}} \mathrm{D}$.

$\mathrm{C}_{\mathrm{II}}=1 \mathrm{C}_{\mathrm{II}} \mathrm{V}$, plus rarement 0,3 plus rarement $4 \mathrm{C}_{\mathrm{II}} \mathrm{L}, 2$ à $4 \mathrm{C}_{\mathrm{II}} \mathrm{D}_{1}$, le plus souvent 3 , et $3 \mathrm{C}_{\mathrm{II}} \mathrm{D}_{2}$.

$\mathrm{C}_{\text {III }}=1 \mathrm{C}_{\text {III }} \mathrm{V}, 6$ à $8 \mathrm{C}_{\text {III }} \mathrm{L}$, le plus souvent $7,1 \mathrm{C}_{\text {III }} \mathrm{D}$.

$\mathrm{C}_{\mathrm{IV}}=1 \mathrm{C}_{\mathrm{IV}} \mathrm{D}$. 


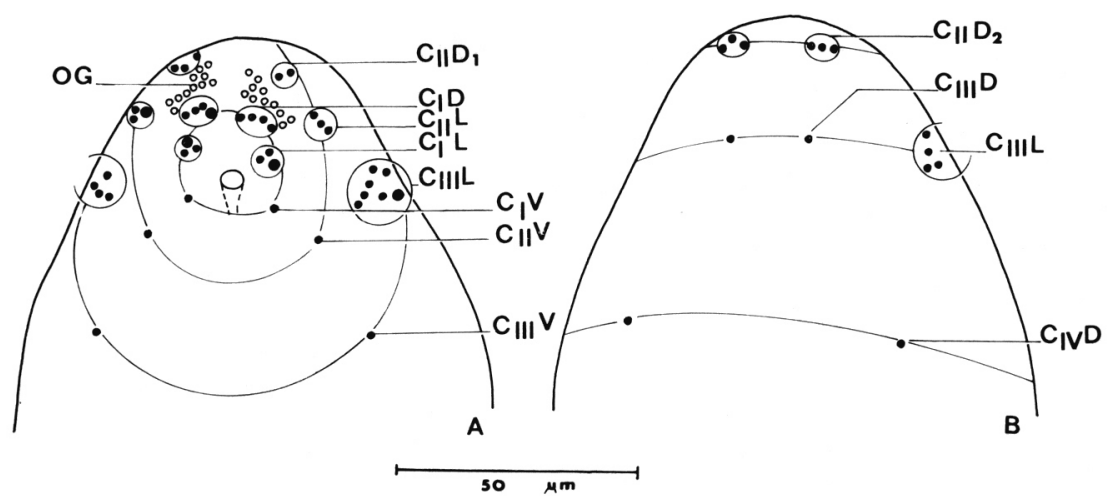

Fig. 2. - Chétotaxie céphalique.

$A$, vue ventrale; $B$, vue dorsale (O. G. : orifices des glandes de pénétration).

Outre ces éléments sensoriels, le nitrate d'argent imprègne pour chaque hémicorps une dizaine d'orifices de glandes désignés par O. G. sur la figure.

$b$ - La région Corporelle (fig. $3 A$ et $B$ )

De part et d'autre du plan médio-sagittal :

$$
\begin{aligned}
& \mathrm{A}_{\mathrm{I}}=1 \mathrm{~A}_{\mathrm{I}} \mathrm{L}, 1 \text { à } 3 \mathrm{~A}_{\mathrm{I}} \mathrm{D} \text { le plus souvent } 2 . \\
& \mathrm{A}_{\mathrm{II}}=1 \mathrm{~A}_{\mathrm{II}} \mathrm{D} . \\
& \mathrm{P}_{\mathrm{I}}=1 \mathrm{P}_{\mathrm{I}} \mathrm{L} . \\
& \mathrm{P}_{\mathrm{II}}=1 \mathrm{P}_{\mathrm{II}} \mathrm{L} . \\
& \mathrm{P}_{\mathrm{III}}=1 \mathrm{P}_{\mathrm{III}} \mathrm{V}, 1 \mathrm{P}_{\mathrm{III}} \mathrm{L} .
\end{aligned}
$$

Nous n'avons vu ni papille $\mathrm{A}_{\mathrm{III}}$, ni papille $\mathrm{M}$.

\section{$c$ - L’acétabulum (fig. 3 C)}

Il porte un total de 8 papilles réparties sur 3 cycles :

$$
\mathrm{S}=1 \mathrm{~S}_{\mathrm{I}}, 5 \mathrm{~S}_{\mathrm{II}}, 2 \mathrm{~S}_{\mathrm{III}} \text {. }
$$

$d$-- La région caudale ( $f i g .3 D$ et $E$ )

- La zone d'attache au tronc caudal porte un anneau de 6 à 7 papilles, suivi de 2 papilles.

- Le tronc caudal porte :

- deux rangées médio-dorsales portant chacune : 23 à 27 papilles UD ;

- deux rangées médio-ventrales portant chacune : 29 à 33 papilles UV;

- deux rangées dorso-latérales UDL préfurcales portant chacune : 9 à 11 papilles ;

- deux rangées ventro-latérales UVL préfurcales portant chacune : 10 à 13 papilles. 

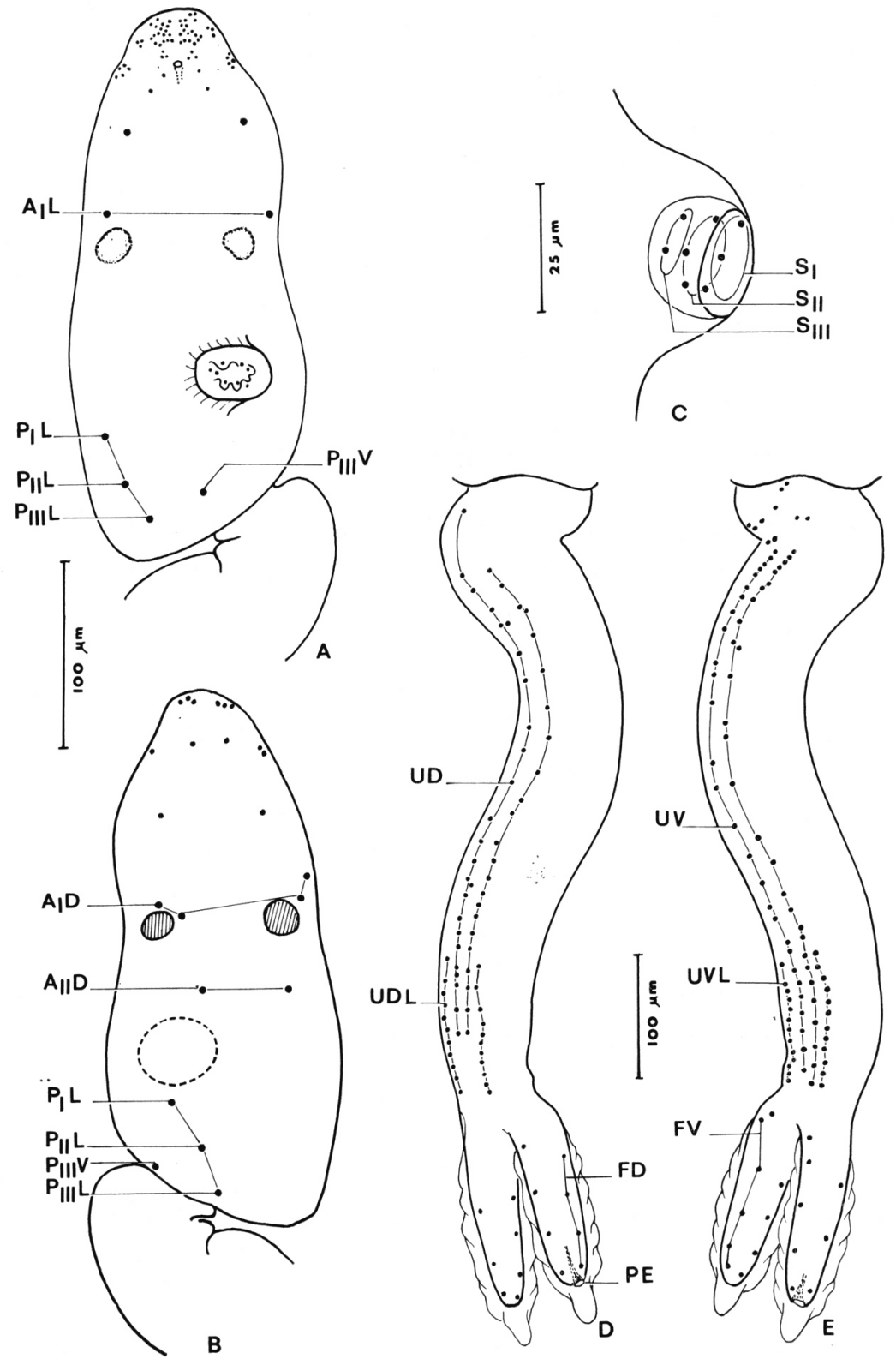

FIG. 3. - Chétotaxie. A : corps cercarien, vue ventro-latérale. B : corps cercarien, vue dorsolatérale. C : acétabulum. D : queue, vue dorso-latérale. F : queue, vue ventro-latérale (PE : Pore Excréteur). 
- Les fourchons : chaque fourchon porte 15 à 18 papilles, soit 3 à 4 paires de ventro-latérales $\mathrm{FV}$, alternant avec 3 à 4 paires de dorso-latérales FD; la partie proximale du fourchon porte en outre une paire de papilles ventro-latérales.

Le nitrate d'argent imprègne les pores excréteurs (désignés par P. E. sur la figure) et la portion distale des canaux excréteurs furcaux.

\section{Discussion}

Dans la systématique classique, les Spirorchiidae appartiennent aux Strigeata. Parmi ceux-ci les Spirorchiidae, les Clinostomidae et les Sanguinicolidae ont des cercaires pourvues d'une nageoire ou d'une crête dorsale.

La chétotaxie des cercaires de Strigeata a fait l'objet des travaux de différents auteurs récapitulés par Bayssade-Dufour (1979), auxquels s'ajoutent les descriptions de Khalifa (1974), Niewiadomska (1980), Richard (1982), Niewiadomska et Moczon (1982), Simon Martin et Rojo Vazquez (1984) et Niewiadomska (1986).

Chez notre cercaire, seuls les cycles $\mathrm{C}_{\mathrm{I}}, \mathrm{C}_{\mathrm{II}}$ et $\mathrm{C}_{\text {III }}$ sont complets, c'est-à-dire qu'ils possèdent des soies $\mathrm{V}$, $\mathrm{L}$ et $\mathrm{D}$. Le cycle $\mathrm{C}_{\text {IV }}$ est dépourvu de soies $\mathrm{V}$ et $\mathrm{L}$. Les cycles $\mathrm{A}_{I}, \mathrm{~A}_{I I}, \mathrm{P}_{\mathrm{I}}, \mathrm{P}_{\text {II }}$ sont dépourvus de soies $\mathrm{V}$; les cycles $\mathrm{P}_{\mathrm{I}}, \mathrm{P}_{\text {II }}$ et $\mathrm{P}_{\text {III }}$ sont dépourvus de soies D. Cette raréfaction des soies s'observe aussi chez les Clinostomidae et les Sanguinicolidae; il est actuellement difficile de préciser si cela est le résultat d'une adaptation convergente, due à la présence de la nageoire ou de la crête dorsale, ou si elle révèle une réelle parenté.

L'acétabulum de notre cercaire a trois cycles; c'est la première fois que cette disposition est constatée chez une furcocercaire de Strigeata, où l'acétabulum, lorsqu'il est présent, porte 1 ou 2 cycles de sensilles.

Le tronc caudal porte en moyenne 150 papilles (140 à 177); il diffère de celui de toutes les cercaires de Strigeata actuellement décrites, qui montrent 10 à 75 papilles selon les familles (Bilharziellidae : 10 à 20 ; Schistosomatidae : 18 à 40 ; Clinostomidae : 25 à 30 ; Sanguinicolidae : 20 à 70 ; Strigeidae : 45 à 65 ; Diplostomidae : 60 à 65 ; Cyathocotylidae : 65 à 75).

Il est peu différent de celui d'une cercaire d'Azygiata Transversotrematidae : Transversotrema patialense, qui compte 110 à 135 papilles (Logoreci, 1978).

Les fourchons caudaux portent chacun 15 à 18 papilles ; ils diffèrent totalement de ceux des Sanguinicolidae, Schistosomatidae, Bilharziellidae et Clinostomidae, qui portent chacun 1 à 6 papilles. Ils sont plus proches de ceux des Strigeidae (10 à 30 papilles), et de ceux des Diplostomidae, Cyathocotylidae et Transversotrematidae (20 à 25 papilles).

La chélotaxie de notre cercaire semble donc indiquer que les Spirorchiidae constituent une famille très originale.

Skrjabin (1951) les plaçait avec les Sanguinicolidae dans la superfamille des Sanguinicoloidea. 
La Rue (1957) les regroupait, avec les Sanguinicolidae et les Schistosomatidae, dans celle des Schistosomatoidea. La chétotaxie ne confirme pas cette proximité.

Selon Bayssade-Dufour (1979), l'augmentation du nombre de cycles acétabulaires est un caractère évolué. La présence de trois cycles au niveau de l'acétabulum de la cercaire étudiée indiquerait que celle-ci est l'une des formes les plus évoluées des Strigeata; plus évoluée, en particulier, que celles des Schistosomes, qui ne possèdent que deux cycles acétabulaires.

Cette hypothèse n'est pas en accord avec celles formulées par différents auteurs (Stunkard, 1923 ; Byrd, 1939 ; Mehra, 1950...), pour lesquels les Schistosomes dériveraient des Spirorchiidae.

Il est actuellement difficile de tirer des conclusions définitives sur les analogies observées entre la queue de notre cercaire de Spirorchiidae et celle de Transversotrema patialense :

- d'une part parce que le groupe des Transversotrematidae est très petit, réduit à quelques espèces, que La Rue rattache aux Azygiata; Skrjabin (1958) et Yamaguti (1971 et 1975) ne lui attribuent de position ni dans une superfamille, ni dans un sous-ordre;

- d'autre part, parce que la description de Transversotrema, même si elle se révèle très utile, semble fragmentaire étant donné l'absence notamment de chétotaxie céphalique.

Il est impossible de généraliser à l'ensemble des Spirorchiidae les données acquises avec cette seule espèce de cercaire. Dans tous les cas, celle-ci est intéressante car elle indique une grande originalité vis-à-vis des autres Strigeata et peut être une certaine affinité avec les Azygiata.

\section{RÉFÉRENCES}

Albaret J.-L. : Imprégnation des formations ciliaires de l'appareil excréteur des cercaires par le protéinate d'argent. C. R. Acad. Sci., 1973, sér. D, 276, 1453-1454.

Bayssade-Dufour Ch. : L'appareil sensoriel des cercaires et la systématique des Trématodes digénétiques. Mém. Mus. natn. Hist. nat., 1979, Nelle sér., sér. A, Zool., 113, 81 p.

Bourgat R., Kulo S. D. : Hapalorhynchus tchalimi n. sp. (Digenea) premier Spirorchiidae de tortue d'eau douce en Afrique. Rev. Zool. Afr., 1987, 100, 435-441.

Byrd E. E. : Studies on the blood-flukes of the family Spirorchiidae. Part II. Revision of the family and description of new species. J. Tenn. Acad. Sci., 1939, 14, 116-161.

Combes C., Bayssade-Dufour Ch., Cassone J. : Sur l'imprégnation et le montage des cercaires pour l'étude chétotaxique. Ann. Parasitol. Hum. Comp., 1976, 51, 399-400.

Goodman J. D. : A new blood fluke, Hapalorhynchus beadlei n. sp. (Spirorchiidae), and a note on Allossostomoides [sic] (Paramphistomidae), in Pelusios williamsi lutescens from Uganda. Trans. amer. Micros. Soc., 1987, 106, 80-84.

Justine J.-L. : Étude ultrastructurale comparée de la spermiogenèse des Digènes et des Monogènes (Plathelmintes). Relation entre la morphologie du spermatozoïde, la biologie de la fécondation et la phylogénie. Thèse Doct. d'État, Univ. Montpellier, 1985, $230 \mathrm{p}$.

Kralifa R. : Studies on Schistosomatidae Looss, 1899 (Trematoda) of aquatic birds of Poland: II. Gigantobilharzia mazuriana sp. n. with a discussion of the sub family Gigantobilharziinae Mehra, 1940. Acta Parasit. polon., 1974, 22, 265-284.

La Rue G. R. : The classification of Digenetic Trematoda: a review and a new system. Expl. Parasit., 1957, 6, 306-344.

Logoreci P. : Documents inédits en communication personnelle. 1978. 
Looss A. : Weitere Beiträge zur Kenntnis der Trematoden-Fauna Aegyptens, zugleich versuch einer natürlichen Gliederung des Genus Distomum Retzius. Zool. Jb. Abt. Syst., 1899, 12, $521-784$.

Menra H. R. : Evolution of the blood-flukes and strigeid trematcdes. Presidential address 19th Ann Meet. Natn. acad. Sci. India (Jan. 22nd 1950), 38 p.

Nassi H. : Spirorchiidae in Combes C., coordinateur, Albaret J.-L., Arvy L., Bartoli P., Bayssade-Dufour Ch., Deblock S., Durette-Desset M.-C., Gabrion C., Jourdane J., Lambert A., Léger N., Maillard C., Matricon M., Nassi H., Prévot G., Richard J., Théron A. : Atlas mondial des cercaires. Mém. Mus. natn. Hist. nat., 1980, Nelle sér., sér. A, Zool., 235 p.

Niewiadomska K. : The cercaria of Cyathocotyle bithyniae Sudarikov, 1974 (Trematoda: Cyathocotylidae). Acta Parasit. polon., 1980, 26, 137-142.

Niewiadomska K., Moczon T. : The nervous system of Diplostomum pseudospathaceum Niewiadomska (Digenea: Diplostomidae). I. Nervous system and chaetotaxy in the cercaria. Z. Parasitenkde, 1982, 68, 295-304.

Niewiadomska K. : Verification of the life-cycles of Diplostomum spathaceum (Rudolphi, 1819) and D. pseudospathaceum Niewiadomska, 1984 (Trematoda, Diplostomidae). Syst. Parasitol., $1986,8,23-31$.

Richard J.: Répartition des structures argyrophiles chez deux cercaires de Strigéidés : Cotylurus brevis Dubois et Rausch, 1950 et Apatemon (A.) minor Yamaguti, 1933. Ann. Parasitol. Hum. Comp., 1982, 5\%, 453-465.

Simon Martin F., Rojo VazQuez F. A. : Nota previa sobre la distribution de las papilas sensoriales de la cercaria de Sanguinicola sp. (Salamanca, Espana). Rev. Iber. Parasitol., 1984, $44,99-100$.

Skruabin K. I. : Les Trématodes de l'animal et de l'homme. Akad. Nauk. SSSR, 1951, V, 624 p. (En russe).

Smith J. W. : The blood Flukes (Digenea: Sanguinicolidae and Spirorchiidae) of Cold-blooded Vertebrates and some Comparison with the Schistosomes. Helminth. Abstr., 1972, sér. A, $41,161-204$.

Stunkard H. W. : Studies on North American blood flukes. Bull. Amer. Mus. Nat. Hist., 1923, $48,165-221$.

Yamaguti S. : Synopsis of Digenetic Trematodes of Vertebrates. I-II. 1971, Keigaku Publishing Co., Tokyo, 1074 p.

Yamagut S. : A synoptical review of life histories of Digenetic Trematodes of Vertebrates. 1975, Keigaku Publishing Co. Ltd., Yugaku Sha Ltd., 590 p. 